\title{
The endocannabinoid system in modulating fear, anxiety, and stress
}

\author{
Rafael Maldonado, MD, PhD; David Cabañero, DVM, PhD; Elena Martín-García, PhD
}

The endocannabinoid system is widely expressed in the limbic system, prefrontal cortical areas, and brain structures regulating neuroendocrine stress responses, which explains the key role of this system in the control of emotions. In this review, we update recent advances on the function of the endocannabinoid system in determining the value of fear-evoking stimuli and promoting appropriate behavioral responses for stress resilience. We also review the alterations in the activity of the endocannabinoid system during fear, stress, and anxiety, and the pathophysiological role of each component of this system in the control of these protective emotional responses that also trigger pathological emotional disorders. In spite of all the evidence, we have not yet taken advantage of the therapeutic implications of this important role of the endocannabinoid system, and possible future strategies to improve the treatment of these emotional disorders are discussed.

(c) 2020, AICH - Servier Group

Dialogues Clin Neurosci. 2020;22(3):229-239. doi:10.31887/DCNS.2020.22.3/rmaldonado

Keywords: amygdala; 2-arachidonoylglycerol; anandamide; $\mathrm{CB}_{1}$ receptor; $\mathrm{CB}_{2}$ receptor; emotional response; HPA axis; limbic system; THC

\section{Introduction}

Anxiety disorders are the most frequent mental alterations worldwide in different socioeconomic regions and are linked with significant comorbidities. ${ }^{1}$ Fear and worry are core symptoms of the entire spectrum of anxiety disorders in the Diagnostic and Statistical Manual of Mental Disorders, $5^{\text {th }}$ Edition (DSM-5). ${ }^{2}$ These disorders have a complex and multifactorial origin resulting from the interaction of multiple genes and environmental factors that lead to large interindividual variability. Chronic stress is one of the main environmental risk factors that disrupt coping circuits and contributes to the etiopathology of these psychiatric disorders. In contrast, exposure to mild stress may act as a protective factor by improving coping strategies in future stressful events. ${ }^{3}$ The reasons why some individuals exposed to similar chronic stress are vulnerable and develop anxiety disorders whereas others are resilient remain unknown. Importantly, there is a lack of successful treatments for these disorders, revealing an urgent need for a better understanding of their neurobiological mechanisms.

Remarkable progress has recently been made in understanding the circuitries underlying anxiety disorders. ${ }^{3} \mathrm{~A}$ large amount of this neurobiological research has been focused on networks targeting the limbic and the hypothalamic-pituitary-adrenal (HPA) axis and its modulation by different neurotransmitters, with special emphasis on the endocannabinoid system. The endocannabinoid system is extensively distributed in the central nervous system (CNS) and plays a crucial role in the control of emotional responses. ${ }^{4}$ Thus, cannabis users identify a subjective sensation of relaxation after consumption, which has been linked

Author affiliations: Laboratory of Neuropharmacology-Neurophar, Department of Experimental and Health Sciences, Universitat Pompeu Fabra (UPF), Barcelona, Spain (Rafael Maldonado, David Cabañero, Elena Martín-García); Hospital del Mar Medical Research Institute (IMIM), Barcelona, Spain (Rafael Maldonado). Address for correspondence: Professor R. Maldonado, Laboratory of Neuropharmacology, Universitat Pompeu Fabra, Dr Aiguader, 88, Barcelona 08003, Spain (email: rafael.maldonado@upf.edu) 


\section{Original article}

The endocannabinoid system in fear, anxiety, and stress - Maldonado et al

with the role of the endocannabinoid system in modulating fear, anxiety, and stress responses.

The endocannabinoid system is a crucial modulatory system allowing the organism to adapt to its changing environment. ${ }^{5}$ This system is integrated by two main G-protein-coupled receptors, cannabinoid 1 receptor $\left(\mathrm{CB}_{1} \mathrm{R}\right)$ and cannabinoid 2 receptor $\left(\mathrm{CB}_{2} \mathrm{R}\right)$, the endogenous ligands that bind to these receptors, mainly anandamide and 2-arachidonoylglycerol (2-AG), and the enzymes responsible for their synthesis and degradation (Figure 1). $\mathrm{CB}_{1} \mathrm{R}$ is highly expressed in the CNS, in neurons, glia, and different cellular organelles such as endosomes and mitochondria. In contrast, $\mathrm{CB}_{2} \mathrm{R}$ is mainly expressed in peripheral cells of the immune system, although it is also present in the CNS, mostly restricted to the microglia. ${ }^{4}$

Here, we discuss recent advances in the role of the endocannabinoid system in determining the value of fearevoking stimuli and promoting appropriate behavioral responses for stress resilience. We review the alterations in endocannabinoid activity during fear-, stress-, and anxiety-related dysfunctions. Fear and anxiety neurobiological substrates mostly overlap and interact with the neurocircuitry that governs the stress response. The concept of stress used in this review will be specific to the stress reaction and will not involve heterogeneous concepts such as subjective distress. ${ }^{6}$ This stress is directly related to the activation of the limbic-HPA axis and stress hormones such as corticotropin-releasing hormone $(\mathrm{CRH})$, adrenocorticotropic hormone (ACTH), and glucocorticoids, a circuit also highly relevant for fear and anxiety. Links among the limbic system, fear circuits, and treatments for worry and fear across the spectrum of anxiety disorders are discussed throughout this review. Understanding the neurobiological mechanisms underlying resilience versus vulnerability to develop anxiety disorders is expected to open novel therapeutic interventions to battle these disorders.

\section{Endocannabinoid system and fear behavior}

Fear is the emotional response to real or perceived imminent threats, whereas anxiety is the anticipation of future threat. Fear is a normal emotion when it appears under a context of the threat, and it is considered an important part of the evolutionary reaction of survival that prepares

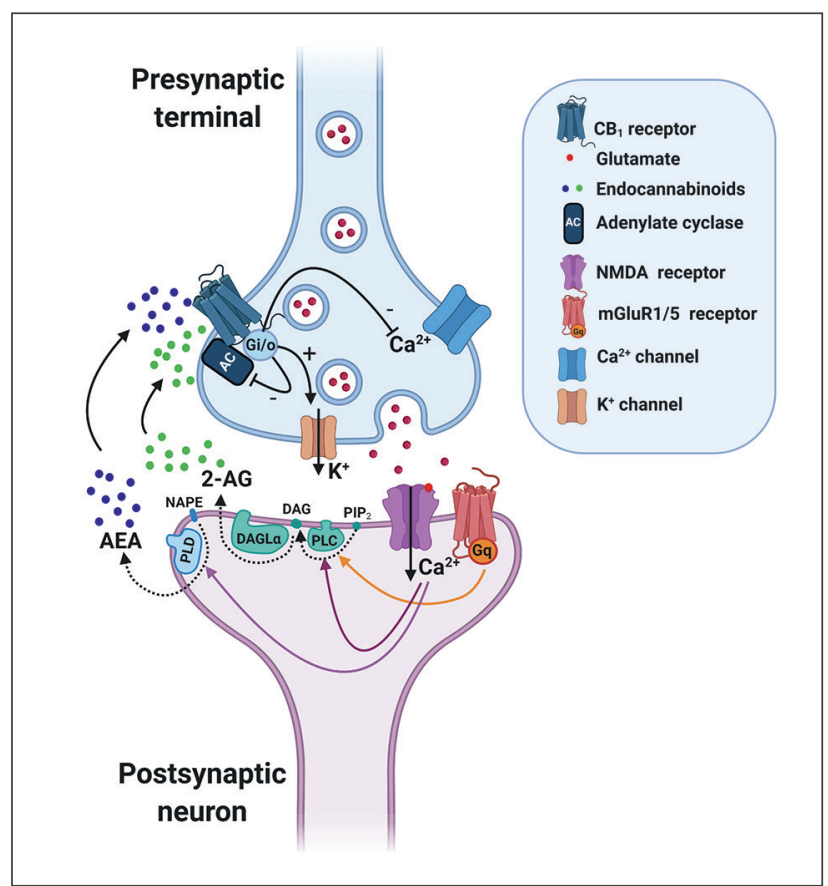

Figure 1. Overview of the endocannabinoid-mediated synaptic signaling at glutamatergic terminals. The endocannabinoid system is a retrograde neuromodulator. After neuronal depolarization at the postsynaptic level, the endocannabinoids are synthesized on demand and travel backwards to bind the presynaptic cannabinoid 1 receptor $\left(C_{1} R\right)$. Through this synaptic mechanism, the endocannabinoid system controls different pathophysiological processes including fear, stress, and anxiety. In detail, glutamate released from presynaptic terminals stimulates both ionotropic and metabotropic glutamate receptors, such as N-methyl-D-aspartate (NMDA) and metabotropic glutamate receptor 1 and 5 (mGluR1/5) receptors, leading to postsynaptic depolarization through $\mathrm{Ca}^{2+}$ entry and Gq-protein activation. Increased intracellular $\mathrm{Ca}^{2+}$ concentration stimulates endocannabinoid synthesis through phospholipase C (2-arachidonoylglycerol, 2-AG) and phospholipase D (anandamide, AEA). 2-AG synthesis is also mediated by Gq-protein activation. Endocannabinoids are released to the synaptic cleft and activate presynaptic $C B_{1} R$. C $B_{1} R$ activation by the endocannabinoids $A E A$ and 2-AG triggers several intracellular pathways via $\mathrm{Gi} /$ o proteins, including inhibition of adenylate cyclase (AC) activity, membrane hyperpolarization through activation of $\mathrm{K}^{+}$channel currents and blockage of $\mathrm{Ca}^{2+}$ voltage-dependent channels, which results in the inhibition of glutamate release. Abbreviations: 2-AG, 2-arachidonoylglycerol; AC, adenylate cyclase; $\mathrm{AEA}$, anandamide; $\mathrm{CB}_{1}$, cannabinoid 1 receptor; DAG, diacylglycerol; DAGL $\alpha$, diacyl glycerol lipase $\alpha$; mGluR1/5; metabotropic glutamate receptor 1 and 5; NAPE, N-arachidonoyl-phosphatidyl ethanol amine; NMDA, $\mathrm{N}$-methyl-D-aspartate; PLC, phospholipase C; PLD, phospholipase D; PIP2, phosphatidyl inositol bisphosphate 


\section{Original article}

The endocannabinoid system in fear, anxiety, and stress - Maldonado et al

to fight or escape. Appropriate fear responses are adaptive and allow the individual to cope with dangerous or stressful situations, but exaggerated fear responses can lead to anxiety disorders. ${ }^{2}$ Although fear and anxiety can overlap, they also differ. Thus, fear is often associated with surges of autonomic arousal necessary for a fight, thoughts of immediate danger, and escape behaviors, whereas anxiety is more often associated with muscle tension and caution in preparation for future danger and with alert or avoidance behaviors. $^{7}$

The amygdala is a limbic hub of communications crucial for fear behavior owing to its anatomical connections integrating sensory and cognitive information (Figure 2). The emotion of fear may be controlled through reciprocal

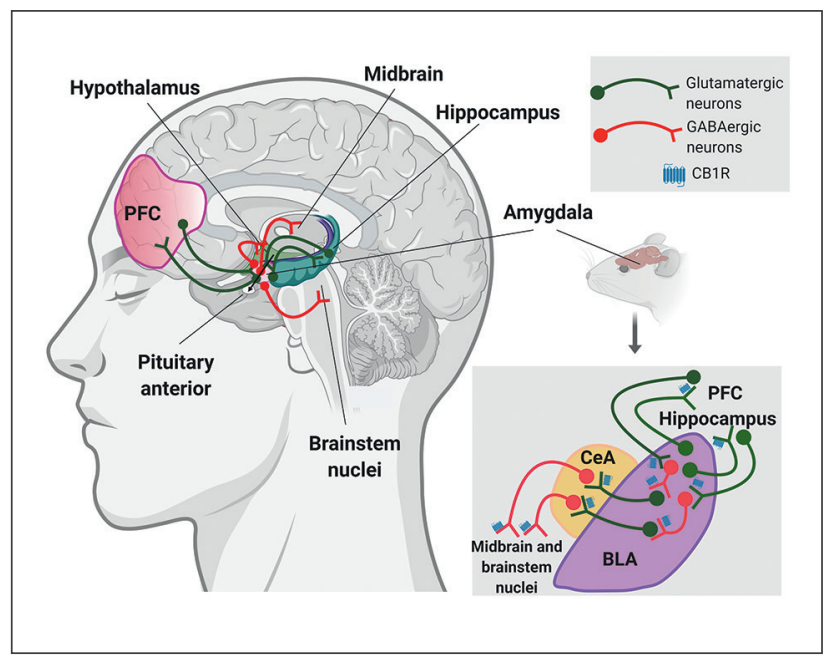

networks with key areas of prefrontal cortex (PFC). Motor responses of fear, mainly fighting and escape, are regulated by connections between the amygdala and periaqueductal gray. Endocrine responses that complement fear are mainly mediated by connections between amygdala and hypothalamus, leading to changes in the HPA axis. ${ }^{7}$ The autonomic nervous system is also stimulated in response to fearful stimuli, activating the cardiovascular system (enhanced blood pressure and heart rate) through increased connectivity between amygdala and locus ceruleus, the major source of brain noradrenergic innervation. ${ }^{8}$

Fear and anxiety disorders can also be elicited internally from traumatic memories stored in the hippocampus and reactivated by the amygdala. In posttraumatic stress disorder (PTSD), persistent, recurring memories of traumatic events occur, which the individual is unable to extinguish. ${ }^{3}$ Extinction plays a crucial role and is considered an active associative-learning process. ${ }^{9}$ In normal conditions, natural stimuli that signal threat provoke an innate fear response. This response is used in the fear conditioning paradigm to study fear-based disorders based on Pavlovian conditioning. Such an associative learning process consists of pairing a neutral conditioned stimulus (CS) with an aversive unconditioned stimulus (US) that produces a conditioned fear response (freezing in rodents, skin conductance response in humans). In PTSD patients, fear learning, specifically extinction, is thought to be impaired. Animal models of pathological fear learning are essential to find effective treatments for such disorders. In this context, the endocannabinoid system plays

Figure 2. The amygdala is highly interconnected with the main brain regions involved in fear, stress, and anxiety. These areas include the amygdala, prefrontal cortex, hippocampus, midbrain, brainstem nuclei, pituitary anterior, and the hypothalamus and have similar functions in rodents and humans. Left figure: an overview of this interconnected circuit is represented in the human brain. The amygdala has bilateral glutamatergic interconnections with the prefrontal cortex and the hippocampus to associate emotional, cognitive, and executive functions. The amygdala controls the activity of midbrain, brainstem nuclei, and neuroendocrine areas (hypothalamus and pituitary anterior) through GABAergic projections that mediate emotional, motor, and neuroendocrine responses to fear, stress, and anxiety. Right figure: precise modulation of fear expression by cannabinoid signaling in the same circuit targeting the amygdala is represented in the rodent brain at the bottom right panel. In detail, the role of the cannabinoid 1 receptor $(C B, R)$ depends on the specific cell type and brain region where they are expressed. On GABAergic neurons, activation of $\mathrm{CB}_{1} \mathrm{R}$ leads to a decrease in active coping strategies in the fear conditioning paradigm, which may facilitate anxiogenic-like responses, possibly due to a decreased activity of GABAergic interneurons in the basolateral amygdala that produces an activation of glutamatergic neurons projecting to the central amygdala, which results in enhanced activity of the central amygdala. Conversely, the activation of CB1R on glutamatergic terminals in the central amygdala induces a decrease in passive coping strategies in the fear conditioning paradigm facilitating anxiolytic-like responses, possibly due to attenuated glutamatergic excitation from the basolateral amygdala, which results in a decreased activation of GABAergic neurons in the central amygdala that project to the midbrain and other brainstem nuclei. These results point to a supposed bimodal control of fear expression by cannabinoid signaling in amygdala-dependent circuits that can also be modulated by glutamatergic inputs from the prefrontal cortex and the hippocampus. Abbreviations: BLA, basolateral amígdala; $C_{1} R_{\text {, }}$ cannabinoid 1 receptor; CeA, central amygdala; PFC, prefrontal cortex 


\section{Original article}

The endocannabinoid system in fear, anxiety, and stress - Maldonado et al

a crucial role in fear-related brain circuits and is crucially involved in the modulation of fear-memory processing. ${ }^{8}$

The role of the endocannabinoid system in modulating the neurobiological mechanisms involved in fear behavior has been widely investigated. ${ }^{9}$ Two neuronal populations expressing $\mathrm{CB}_{1} \mathrm{R}$ widely distributed thorough the brain have been described: cortical glutamatergic and GABAergic forebrain neurons. The equilibrium between GABAergic and glutamatergic transmission provides an appropriate emotional reactivity in physiological conditions (Figure 2). Under stressful circumstances, the glutamatergic tone increases, producing an imbalance between excitatory and inhibitory transmission. To compensate for this glutamatergic overexcitation, an adaptive compensatory mechanism is activated by $\mathrm{CB}_{1} \mathrm{R}$ downregulation in GABAergic terminals. This downregulation promotes increased GABAergic inhibition of glutamatergic transmission that reestablishes the equilibrium between excitation and inhibition. ${ }^{8}$ The behavior of mutant mice lacking $\mathrm{CB}_{1} \mathrm{R}$ in cortical glutamatergic neurons indicates that the modulatory effect of $\mathrm{CB}_{1} \mathrm{R}$ in these excitatory neurons plays a complementary role in fear extinction. ${ }^{10}$ Therefore, the role of $\mathrm{CB}_{1} \mathrm{R}$ depends on the specific cell type and brain region where they are expressed. Importantly, the deletion of $\mathrm{CB}_{1} \mathrm{R}$ in forebrain GABAergic neurons of GABA-CB $\mathrm{R}$ knockouts leads to an augmentation of active coping strategies in the fear conditioning paradigm, possibly due to an increased inhibition of glutamatergic cells in the basolateral amygdala (BLA), which reduces central amygdala $(\mathrm{CeA})$ excitation. ${ }^{11}$ Conversely, the deletion of $\mathrm{CB}_{1} \mathrm{R}$ in cortical glutamatergic neurons of Glu-CB $\mathrm{R}$ knockouts induces augmentation of passive coping strategies in the fear conditioning paradigm, possibly due to increased glutamatergic excitation in the BLA, which results in CeA hyperactivation. ${ }^{11}$ These results point to a bimodal cannabinoid control of fear in amygdala-dependent circuits $^{12}$ (Figure 2). In agreement, low tetrahydrocannabinol (THC) doses have been proposed to act preferentially at $\mathrm{CB}_{1} \mathrm{R}$ on glutamatergic neurons, whereas high THC doses have been proposed to act also at GABAergic neurons. ${ }^{13}$ $\mathrm{CB}_{1} \mathrm{R}$ is less abundant on cortical glutamatergic neurons, but its activation produces more pronounced effects than on GABAergic neurons. ${ }^{14}$ Furthermore, $\mathrm{CB}_{1} \mathrm{R}$ antagonists microinjected in the BLA impair long-term extinction, and $\mathrm{CB}_{1} \mathrm{R}$ antagonism into the $\mathrm{CeA}$ reduces within-session extinction. ${ }^{15}$ The infralimbic PFC also has an important role in fear extinction, as shown by the impaired fear extinction after $\mathrm{CB}_{1} \mathrm{R}$ blockade in this subregion. ${ }^{16}$

In agreement with these data, mice deficient in diacylglycerol lipase- $\alpha$ (DAGL $\alpha$ ), the enzyme involved in 2-AG synthesis, have reduced $2-\mathrm{AG}$ brain levels and display a phenotype related to impaired fear extinction. ${ }^{17}$ In contrast, pharmacological inhibition of monoacylglycerol lipase (MAGL), the enzyme involved in 2-AG degradation, enhances 2-AG levels and impairs fear extinction via $\mathrm{CB}_{1} \mathrm{R}$ of GABAergic neurons. ${ }^{17}$ These results suggest that an optimal level of $2-\mathrm{AG}$ is needed for appropriate processing of fear responses and that altered 2-AG levels impair the extinction process. ${ }^{5}$ With respect to anandamide, inhibition of the enzyme involved in its degradation, fatty acid amide hydrolase (FAAH) in the BLA, facilitates fear extinction by $\mathrm{CB}_{1} \mathrm{R}$ activation. ${ }^{18} \mathrm{Re}$-exposure to the $\mathrm{CS}$ alone increases the activity of the endocannabinoid system to promote extinction, and the fear response is reduced after $\mathrm{CB}_{1} \mathrm{R}$ activation. ${ }^{5}$ Therefore, the endocannabinoid system is crucial for appropriate fear extinction.

On the other hand, chronic stress can have deleterious effects regarding the extinction of conditioned fear, which may interfere in the treatment of PTSD patients. It is hypothesized that chronic stress creates a hypocannabinergic state resulting in impaired fear extinction that can be alleviated by $\mathrm{CB}_{1} \mathrm{R}$ agonists. ${ }^{5}$ Chronic stress can produce a switch from passive to active coping strategies, and constitutive $\mathrm{CB}_{1} \mathrm{R}$ deletion disrupts this adaptation favoring passive responses that seem to depend on $\mathrm{CB}_{1} \mathrm{R}$ expressed on cortical glutamatergic neurons. ${ }^{11}$ Conversely, $\mathrm{CB}_{1} \mathrm{R}$ expressed on forebrain GABAergic neurons are related to coping strategies because mice lacking $\mathrm{CB}_{1} \mathrm{R}$ in these neurons display predominantly active behaviors. ${ }^{11}$ Importantly, when the responses to CS are measured during a long-term period of CS presentation, no differences in the quantity of fear responses are observed 


\section{Original article}

The endocannabinoid system in fear, anxiety, and stress - Maldonado et al

in these conditional mutant mice, suggesting that $\mathrm{CB}_{1} \mathrm{R}$ in cortical glutamatergic or forebrain GABAergic neurons does not affect the memory of the conditioning event, but determines the coping style of an individual toward the threat. $^{5}$

\section{Endocannabinoid system and stress}

Stress is an alteration of homeostasis as a consequence of external or internal threats. Indeed, acute stressors elicit immediate and protracted neuroendocrine responses with protective effects. These responses involve the activation of the sympathetic nervous system and the HPA axis. Within seconds of stress exposure, noradrenaline and adrenaline are released through sympathetic postganglionic neurons and adrenal gland chromaffin cells contributing to fight-orescape protective responses. In parallel, the HPA axis is activated through $\mathrm{CRH}$ release from the hypothalamus, which leads to ACTH release from the pituitary gland. ACTH in the general blood stream reaches adrenal glands that pour glucocorticoids into the blood circulatory system. These corticoids activate glucocorticoid receptors that increase glucose availability and trigger transcriptional changes partly directed to limit inflammation and repair processes that can be postponed.

The endocannabinoid system present in the HPA axis and the sympathetic nervous system plays a crucial role in regulating stress responses (Figure 3). Early studies showed that repeated THC modified dopamine $\beta$-hydroxylase activity in rodent serum, a measure of sympathetic system activation. ${ }^{19}$ This effect was different depending on the basal status of exposed subjects revealing a complex modulatory role of the endocannabinoid system: THC alleviated sympathetic activation in naive mice but potentiated this response in rodents subjected to immobilization stress. $\mathrm{CB}_{1} \mathrm{R}$ controls peripheral and central adrenaline, and noradrenaline release involved in stress-induced memory impairment, ${ }^{20}$ and the sympathetic nervous system partly mediates the anxiety-like effects observed after $\mathrm{CB}_{1} \mathrm{R}$ blockade. ${ }^{21}$ In regard to the HPA axis, pharmacological and knockout studies demonstrate that $\mathrm{CB}_{1} \mathrm{R}$ activity limits hypothalamic $\mathrm{CRH}$ release. ${ }^{22} \mathrm{CB}_{1} \mathrm{R}$ is also present in the pituitary gland and adrenal cortex cells, where it restricts ACTH and glucocorticoid release, respectively. ${ }^{22}$ On the contrary, glucocorticoids induce fast increases in endocannabinoid synthesis in brain areas that shape the perception of psychological stressors. ${ }^{23}$ These regions include areas involved in cognitive processes such as PFC and hippocampus, and areas related with affective responses such as the amygdala. ${ }^{24}$ Glucocorticoids released after acute stressors activate G-protein membrane receptors in the BLA, promoting a rapid increase in retrograde 2-AG signaling that leads to suppression of GABAergic synaptic inputs onto BLA principal neurons, inducing fast increases in anxiety-like behavior. ${ }^{25}$ These limbic areas are further connected with the hypothalamus to modulate

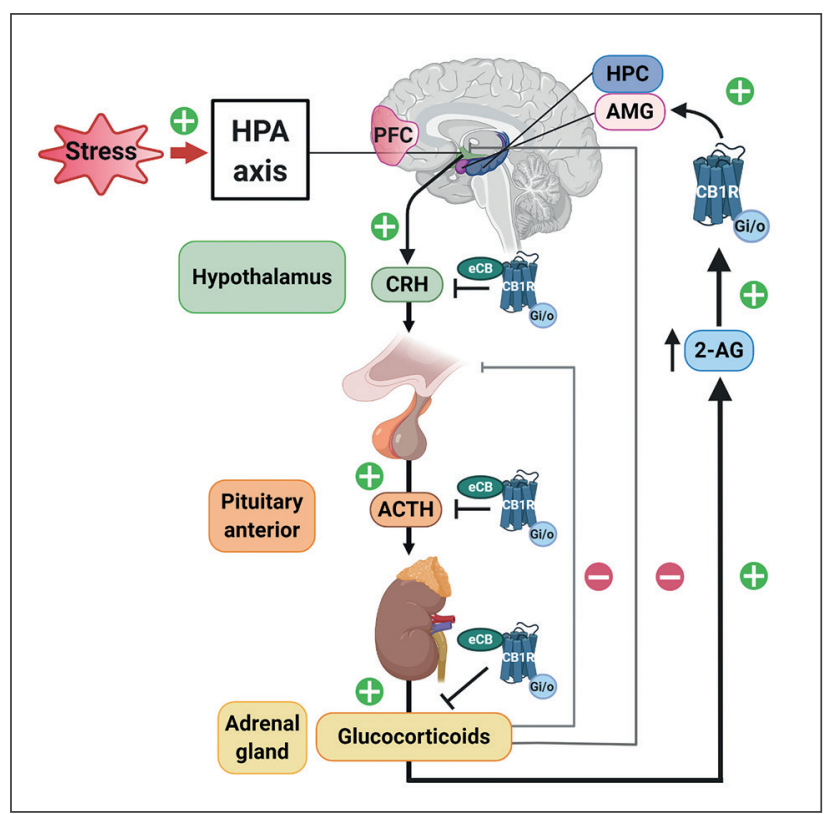

Figure 3. Modulatory effect of the cannabinoid 1 receptor $\left(C_{1} R\right)$ activity in the hypothalamic-pituitary-adrenal (HPA) axis. Under stress conditions, the HPA axis is activated to produce an adaptive defensive response to stress. To counteract excessive HPA-axis activation, $\mathrm{CB}_{1} \mathrm{R}$ activity limits the release of hypothalamic corticotropin-releasing hormone. $C B_{1} R$ is also present in the pituitary gland and adrenal cortex cells, where they restrict adrenocorticotropin hormone and glucocorticoid release, respectively. On the contrary, glucocorticoids induce fast increases in endocannabinoid synthesis in brain areas that shape the perception of stress. These regions include the prefrontal cortex, the hippocampus, and the amygdala. Indeed, glucocorticoids released after acute stressors promote a rapid increase in retrograde 2 -arachidonoylglycerol (2-AG) signaling in these brain areas leading to decreased GABAergic release in the amygdala that induces a fast increase in anxiety-like behavior. Abbreviations: 2-AG, 2-arachidonoylglycerol; ACTH, adrenocorticotropin hormone; $A M G$, amygdala; $C_{1} R$, cannabinoid 1 receptor; $C R H$, corticotropin-releasing hormone; eCB, endocannabinoids; HPC, hippocampus; HPA, hypothalamic-pituitary-adrenal; PFC, prefrontal cortex 


\section{Original article}

The endocannabinoid system in fear, anxiety, and stress - Maldonado et al

stress responses (Figure 3). $\mathrm{CB}_{1} \mathrm{R}$ located in these structures represent a unique opportunity for pharmacological modulation. However, the use of exogenous $\mathrm{CB}_{1} \mathrm{R}$ ligands has been associated with serious health problems, from THC effects promoting addictive behaviors ${ }^{26}$ and anxiety disorders ${ }^{27}$ to the psychiatric alterations (anxiety, depression, or suicidal behaviors) related to the $\mathrm{CB}_{1} \mathrm{R}$ inverse agonist rimonabant. ${ }^{28}$ Therefore, a great deal of research has focused on the pharmacological modulation of endogenous cannabinoids as an alternative approach for the treatment of stress-induced alterations. In this context, the enzymes involved in the synthesis and degradation of endocannabinoids represent potential effective targets with possible better safety profiles than drugs directly acting on $\mathrm{CB}_{1} \mathrm{R}$.

Models of acute immobilization stress provoke immediate increases in FAAH activity, the enzyme involved in anandamide degradation, in amygdala and hippocampus. Enhancing the activity of this degrading enzyme results in glucocorticoid release and stress-related anxiety-like behavior, ${ }^{29}$ whereas FAAH inhibition or costimulation of $\mathrm{CB}_{1} \mathrm{R}$ and $\mathrm{TRPV}_{1}$ (transient receptor potential cation channel subfamily $\mathrm{V}$ member 1) receptors decreases stress-induced corticoid release and anxiogenic behavior., ${ }^{30,31}$ Restraint stress or corticosterone treatments also produce a delayed increase in 2-AG levels in the hypothalamus, PFC, and hippocampus, associated with a decreased HPA-axis activity $^{32}$ that modulates memory processes and pain perception after stressful stimuli. ${ }^{33}$ Therefore, acute stress exposure has emotional and physiological consequences related with decreased anandamide production, but also favors increased 2-AG levels that limit the stress response and have an impact on cognitive performance. ${ }^{33}$

In contrast to acute stress, chronic stress predisposes for the development and aggravation of psychiatric disorders, including anxiety, depression, PTSD, and drug addiction. ${ }^{34}$ Chronic stress induces prominent and sustained changes in the endocannabinoid system, leading to decreases in $\mathrm{CB}_{1} \mathrm{R}$ signaling in brain regions related with emotional processing such as the hippocampus, nucleus accumbens, PFC, dorsal raphe nucleus, hypothalamus, and amygdala. ${ }^{5}$ Indeed, sustained corticosterone increments after repeated restraint stress promote FAAH activity through glucocorticoid stimulation. ${ }^{29}$ As a consequence, anandamide levels are maintained low in the hippocampus, hypothalamus, PFC, and amygdala, ${ }^{35}$ which leads to hyperexcitability and struc- tural remodeling of amygdalar circuits involved in anxiety. Indeed, a rat model of pathological anxiety reveals constitutive increases in corticotropin-releasing factor (CRF) signaling that lead to sustained elevation of FAAH activity and dysregulation of inhibitory control of CeA glutamatergic synapses.$^{36}$ Inhibition of FAAH hyperactivity prevents these effects of chronic stress ${ }^{37}$ and protracted structural and functional changes that chronic stress promotes in BLA glutamatergic neurons. ${ }^{38,39}$ Interestingly, divergent results have been obtained after exposure to other models of chronic stress, ${ }^{40}$ suggesting that the nature of the stress or the existence of previous experiences can lead to differentiated stress-induced neuroadaptations. Indeed, repeated exposure to the same stress provokes increased 2-AG concentrations that underlie habituation to stress in adults. ${ }^{41}$ Further increasing 2-AG levels through blockade of 2-AG degradation prevents the decrease in hippocampal neurogenesis and long-term synaptic effects associated with chronic stress. ${ }^{42}$ Deletion of the 2 -AG synthesizing enzyme DAGL $\alpha$ in PFC strengthens glutamatergic activity in a BLA-PFC-BLA circuit that facilitates stress-induced anxiety-like behavior. ${ }^{43}$ These data show that facilitating synthesis or inhibiting degradation of anandamide and 2-AG have overlapping consequences, suggesting the therapeutic potential of these enzyme modulators for the treatment of stress-related disorders. The redundancy exhibited by $2-\mathrm{AG}$ and anandamide could potentially be exploited to design drug combinations with lower doses of active ingredients and may be useful to tackle different aspects of chronic stress disorders at once.

\section{Endocannabinoid system and anxiety}

Contemporary society is exposed to a multiplicity of external factors that constitute sources of anxiety, including excessive workloads, difficult personal relationships, sexual abuse, drug consumption, digital social media, or even current infectious disease pandemics. ${ }^{44}$ Anxiety can be defined as a mental state of discomfort triggered by uncertain threats. ${ }^{45}$ These threats are estimated to be distant in time or space and lead to anticipatory affective, cognitive, behavioral, and physiological changes and a continuous risk assessment that can persist until the uncertainty is resolved. ${ }^{46}$ Internal changes are adaptive and favor behaviors that protect the organism from dangerous situations, although in certain conditions become disproportional and result in anxiety-related disorders. 


\section{Original article}

The endocannabinoid system in fear, anxiety, and stress - Maldonado et al

One of the main medical reasons for cannabis consumption is anxiety relief, ${ }^{47}$ although anxiogenic responses are also frequently reported after cannabinoid consumption. ${ }^{48}$ Indeed, high doses or long-term treatments with THC induce anxiety in humans and rodents. On the other hand, acute administration of the non-psychotropic cannabinoid cannabidiol (CBD) or low THC doses can relieve anxiety-related behavior. ${ }^{47}$ The Cre-Lox technology allowed the dissection of biological substrates underlying this dual effect of $\mathrm{CB}_{1} \mathrm{R}$ activation. Glutamatergic and GABAergic neurons expressing $\mathrm{CB}_{1} \mathrm{R}$ are widely distributed in key brain areas involved in anxiety, such as the amygdala, hippocampus, or PFC. Presynaptic $\mathrm{CB}_{1} \mathrm{R}$ of cortical glutamatergic neurons inhibit glutamate release, and their activation induces anxiolysis. ${ }^{49}$ In agreement, anxiety-like behavior is observed in mice lacking $\mathrm{CB}_{1} \mathrm{R}$ in cortical glutamatergic neurons, ${ }^{8,50}$ and reinstatement of $\mathrm{CB}_{1} \mathrm{R}$ expression in glutamatergic neurons of $\mathrm{CB}_{1} \mathrm{R}$-knockout mice partly restores normal anxiety levels. ${ }^{51}$ In contrast, $\mathrm{CB}_{1} \mathrm{R}$ activation from the larger population of forebrain GABAergic neurons restricts the inhibitory GABA tone and facilitates anxiety-like behavior in basal conditions. ${ }^{49}$ This function is abrogated in mice lacking $\mathrm{CB}_{1} \mathrm{R}$ in forebrain GABAergic neurons. ${ }^{50,52,53}$ Interestingly, the presence of $\mathrm{CB}_{1} \mathrm{R}$ in forebrain $\mathrm{GABA}$ ergic neurons was also essential for the serenity obtained after physical exercising, interpreted as the opposite of anxiety-like behavior. ${ }^{54}$ Thus, although stimulation of $\mathrm{CB}_{1} \mathrm{R}$ of forebrain GABAergic neurons is related with the anxiogenic effects of high THC doses, the cannabinoid tone of this GABAergic neuronal population can have bidirectional anxiolytic or anxiogenic effects depending on the basal status of the individuals. ${ }^{54}$ This complex modulatory function of the endocannabinoid system on anxiety needs to be carefully understood to allow efficient modulation in the clinics.

Recent advances have started to unravel the complex circuitry involved in the cannabinoid modulation of anxiety. BLA represents a critical brain center for the onset of anxiety in response to environmental cues. Systemic and intra-amygdalar administration of 2-AG and anandamide reuptake inhibitors demonstrate overlapping effects reducing anxiety through $\mathrm{CB}_{1} \mathrm{R}$ expressed in glutamatergic synapses. ${ }^{55-57}$ At least part of this anxiogenic glutamatergic input to the amygdala comes from PFC and hippocampus. Prelimbic PFC glutamatergic neurons are active after threat exposure, as demonstrated by recent optogenetic and cell-specific gene deletion experiments. ${ }^{43} \mathrm{~A}$ traumatic experience (foot-shock) in mice facilitates $2-\mathrm{AG}$ depletion and subsequent enhancement of glutamatergic neurotransmission in this BLA-PFC-BLA pathway to trigger anxiety-like behavior. ${ }^{43}$ The relevance of this circuitry is reflected in clinical studies showing that the anxiolytic effects of THC are associated with reduced activity and disrupted connectivity between these two brain areas. ${ }^{58}$ A persistent weakening of the endocannabinoid tone could lead to enhanced functional BLA-PFC coupling and progression from a physiological stress to an anxiety disorder. The BLA also receives glutamatergic projections from the hippocampus, and these projections are bidirectionally controlled by endocannabinoids. In a similar way, increases in hippocampal 2-AG or anandamide promote stress resilience and anxiolysis, whereas $\mathrm{CB}_{1} \mathrm{R}$ blockade or inhibition of 2-AG production precipitates anxiety. ${ }^{59}$

Local $\mathrm{CB}_{1} \mathrm{R}$ activity has also been associated with anxiogenesis in certain circuits. Thus, the amygdala sends $\mathrm{CB}_{1} \mathrm{R}$ -positive projections to the bed nucleus of the stria terminalis (BNST), a brain region closely involved in anxiety. This area is connected with midbrain structures, including the ventral tegmental area and the locus coeruleus. Glutamatergic and GABAergic projections from the amygdala to BNST are each sufficient for the development of anxious responses to unpredictable stimuli. $\mathrm{CB}_{1} \mathrm{R}$ activity in these projections seems essential for the shift from phasic to sustained fear responses in fear conditioning chambers, interpreted as a reminiscence of the human anxiety against unpredictable threats. ${ }^{60}$ In the context of an established anxiogenic response, blocking cannabinoid activity in the BNST facilitates the transition from persistent to phasic freezing responses, a switch that could allow earlier reinstatement of normal anxious behavior.

A cooperation between the endocannabinoid and monoaminergic systems is required for anxiety modulation. ${ }^{61}$ At a molecular level, heterodimers composed of $\mathrm{CB}_{1} \mathrm{R}$ and $5-\mathrm{HT}_{2 \mathrm{~A}}$ receptors are essential for the effects of $\mathrm{THC}$ promoting anxiolysis and social interaction. ${ }^{61}$ In agreement with this cannabinoid-serotonergic interaction, deleting $\mathrm{CB}_{1} \mathrm{R}$ from serotonergic neurons of the raphe nuclei leads to decreased social interaction and enhanced anxiety-like behavior in a novelty-suppressed feeding test. ${ }^{62}$ Close coordination between both systems has also been reported at a circuit level. $\mathrm{CB}_{1} \mathrm{R}$-expressing glutamatergic neurons of the PFC synapse with serotonergic and GABAergic neurons in the dorsal raphe nuclei. Optogenetic approaches have 


\section{Original article}

The endocannabinoid system in fear, anxiety, and stress - Maldonado et al

revealed that dorsal raphe GABAergic synapses are more sensitive to the inhibitory effect of $\mathrm{CB}_{1} \mathrm{R}$ activity than serotonergic neurons. As a consequence, $\mathrm{CB}_{1} \mathrm{R}$ stimulation provokes a net increase of 5-HT output from the dorsal raphe nuclei. ${ }^{63} \mathrm{In}$ a related circuit, $\mathrm{CB}_{1} \mathrm{R}$ stimulation shows opposite anxiogenic effects controlling the lateral habenula, an area that integrates stimuli from basal ganglia and limbic system and sends output signals to dopamine and serotonergic neurons of the ventral tegmental area ${ }^{64}$ Blocking habenular $\mathrm{CB}_{1} \mathrm{R}$ augments proactive coping behaviors to reduce social stress and diminishes anxiety-like behavior. With cooperation between monoaminergic and endocannabinoid systems starting to be untangled, it will probably constitute an additional source of pharmacological exploitation for anxiety disorders.

Preexisting conditions can modify the effects of the manipulation of the endocannabinoid system on anxiety. Thus, a state of low arousal allows anxiety relief after potentiation of amygdala cannabinoid neurotransmission (2-AG or anandamide), whereas high arousal conditions prevent this effect. ${ }^{33}$ Protracted consequences of cannabinoids have been revealed when administered shortly after threat exposure. Thus, functional magnetic resonance imaging (fMRI) in humans has shown that single THC exposures after threatening stimuli have perceptible effects 1 week later. These effects consist of decreased amygdala-PFC activity and reduced functional coupling among $\mathrm{PFC}$, hippocampus, and dorsal anterior cingulate cortex, a network involved in the extinction of aversive memories. ${ }^{58}$ Thus, the anxiolytic effects of cannabinoids depend on the quality and timing of the threats and could have delayed effects on the expression or extinction of anxiety-related behavior, not detectable immediately after the treatments.

Age and sex of individuals are important determinants for the consequences of cannabinoid exposure on anxiety. ${ }^{65}$ Women present an increased propensity to anxiety-related disorders and biological differences in the endocannabinoid system compared with men. ${ }^{65}$ These sex differences have been studied in animal models of anxiety. $\mathrm{CB}_{1} \mathrm{R}$ knockout males show a basal anxiogenic behavior that is absent in females. ${ }^{66}$ Interestingly, both sexes show anxiogenic responses after $\mathrm{CB}_{1} \mathrm{R}$ blockade, ${ }^{66}$ of higher intensity in adolescent females ${ }^{67}$ However, mice defective in the 2-AG-synthesizing enzyme DAGL $\alpha$ show no gender differences, except for a maternal neglecting behavior inherent to the female condition..$^{17}$ Exposure of pregnant females to $\mathrm{THC}^{68}$ or to polyunsaturated fatty acids required for endocannabinoid synthesis ${ }^{69}$ produces life-long changes on the offspring, altering the distribution and activity of neuronal populations expressing $\mathrm{CB}_{1} \mathrm{R}$. Prenatal THC causes male-specific effects, including disrupted long-term depression (LTD) and heightened excitability in the PFC, enhanced anxiety, and social distancing. ${ }^{68}$ Although the female offspring did not show overt behavioral changes and their PFC LTD was maintained, transcriptional modifications and a switch from $\mathrm{CB}_{1} \mathrm{R}$ to TRPV1-mediated LTD have been found, suggesting the existence of protective mechanisms or latent alterations in females. Further studies will be needed to understand the long-lasting consequences of THC exposure on the developing female brain.

The possible impact of periadolescent cannabinoid exposure on anxiety levels during adulthood has been investigated. Reduced anxiety has been revealed in previously exposed males, although such an anxiolytic effect is inconsistently reported in adult females. ${ }^{70} \mathrm{THC}$ exposure or pharmacological disruption of the endocannabinoid tone during early adolescence also provokes decreased sociability in both sexes, occasionally without evident anxiety alterations, ${ }^{71,72}$ that may not persist in the adult age. ${ }^{71,73}$ However, the existence of significant modifications during a critical age for brain development demands caution with cannabinoid intake. Indeed, fMRI studies in humans have described long-lasting consequences of THC exposure, including increased gray-matter volume of brain temporal areas and amygdala hypersensitivity, associated with the development of anxious symptomatology. ${ }^{74,75}$ The effects of cannabinoid exposure may be different in individuals who suffered previous threatening experiences. Thus, rodents subjected to early life stress show reduced anxiety and cognitive and social impairments after $\mathrm{CB}_{1} \mathrm{R}$ agonist administration during late adolescence. These effects are associated with the normalization of biochemical alterations in BLA, PFC, and hippocampus of males and females ${ }^{65}$ Additional studies will be necessary to evaluate the therapeutic possibilities of cannabinoid treatments under these particularly sensitive conditions.

\section{Concluding remarks}

Recent studies have allowed a better understanding of the crucial role played by the endocannabinoid system in 


\section{Original article}

The endocannabinoid system in fear, anxiety, and stress - Maldonado et al

fear, anxiety, and stress. $\mathrm{CB}_{1} \mathrm{R}$ located in GABAergic and glutamatergic terminals of the limbic system modulate the glutamatergic overexcitation in the amygdala, leading to fear behavior. The endocannabinoid system protects against this overactivation by downregulating $\mathrm{CB}_{1} \mathrm{R}$ in GABAergic terminals, and $\mathrm{CB}_{1} \mathrm{R}$ in glutamatergic terminals also play a complementary role to promote fear extinction. $\mathrm{CB}_{1} \mathrm{R}$ located in the HPA axis and the sympathetic nervous system are key components to modulate acute stress. $\mathrm{CB}_{1} \mathrm{R}$ activation limits HPA axis and sympathetic activation during stress, whereas glucocorticoids induce through the endocannabinoid system the activation of amygdala circuits to promote stress perception. These effects seem mediated by an enhancement of 2-AG levels and a decreased anandamide synthesis in brain areas related to emotional and cognitive responses during stress. In contrast, chronic stress exposure leads to maladaptive changes involving the endocannabinoid system that may predispose to the development of emotional disorders. On the other hand, $\mathrm{CB}_{1} \mathrm{R}$ located in glutamatergic and GABAergic terminals seem to play an opposite role in anxiety control. $\mathrm{CB}_{1} \mathrm{Rs}$ in cortical glutamatergic terminals inhibit glutamate release, and their activation induces anxiolysis, whereas $\mathrm{CB}_{1} \mathrm{R}$ activation from the larger population of forebrain GABAergic neurons restricts the inhibitory GABA tone and mainly facilitates anxiety. This opposite role of different $\mathrm{CB}_{1} \mathrm{R}$ leads to complex endocannabinoid circuits to regulate anxiety, where increasing the endocannabinoid activity is mostly related with anxiolysis, although an enhanced anxiety is also mediated in particular circuits. Therefore, the endocannabinoid system represents an excellent target for therapeutic purposes in emotional disorders, and the main medical reason for cannabis consumption is anxiety relief. As expected, considering the complexity of the endocannabinoid role in anxiety, anxiogenic responses are also reported after cannabis consumption together with multiple side effects, which limits the use of these cannabinoid agonists for treating emotional disorders. Indeed, although self-reports of cannabis users argue acute anxiolytic consequences, the effects of chronic consumption are controversial and systematic studies are still missing. ${ }^{76}$ Indeed, chronic cannabis use has been reported to produce opposite effects in some individuals, raising the risk for the onset of anxiety disorders and the consequent enhancement in the severity of negative emotional symptoms. ${ }^{76}$ Furthermore, even though chronic cannabis use does not produce clear manifestations of physical dependence, abruptly stopping its use can trigger withdrawal symptoms, such as sleep disturbance, irritability, and anxiety. ${ }^{26}$ In summary, additional studies are needed to determine the possible beneficial effects of modulating the activity of the endocannabinoid system for therapeutic purposes on fear, anxiety, and stress. ${ }^{77}$

\section{Future directions}

In spite of the prominent role of the endocannabinoid system in emotional disorders, we have not yet taken advantage of the therapeutic implications of this important pathophysiological mechanism. The endocannabinoid system is very widely expressed in the CNS and a systemic modulation of its activity with classical ligands may not be the best pharmacological approach due to the large number of brain circuits that are affected. Indeed, important side effects have been reported when the activity of the endocannabinoid system is enhanced with agonists, such as THC, or decreased with inverse agonists, such as rimonabant. The use of compounds able to modify the synthesis/degradation/reuptake of endocannabinoids ${ }^{78}$ or biased modulators of cannabinoid receptor activity ${ }^{79}$ could certainly provide a more appropriate strategy to obtain pharmacological responses restricted to a beneficial effect on emotional disorders. Preclinical data and preliminary findings in humans obtained with these compounds suggest that indirect agonists and biased cannabinoid modulators could represent novel pharmacological tools to obtain therapeutic benefits by modifying the activity of the endocannabinoid system.

Acknowledgments/Disclosures: The authors have no conflict of interest. This work was supported by the Spanish Ministerio de Economía y Competitividad-MINECO (\#SAF2017-84060-R-AEI/FEDER-UE), the Spanish Instituto de Salud Carlos III, RETICS-RTA (\#RD12/0028/0023), the Generalitat de Catalunya, AGAUR (\#2017-SGR-669), ICREA-Acadèmia (\#2015) and the Spanish Ministerio de Sanidad, Servicios Sociales e Igualdad, Plan Nacional Sobre Drogas of the Spanish Ministry of Health (\#PNSD2017I068) (RM); Fundació La Marató-TV3 (\#2016/20-30) and Plan Nacional Sobre Drogas of the Spanish Ministry of Health (\#PNSD-2019I006) (EMG). Figures were created with BioRender.com. 


\section{Original article}

The endocannabinoid system in fear, anxiety, and stress - Maldonado et al

\section{References}

1. Stein DJ, Scott KM, de Jonge P, Kessler RC. Epidemiology of anxiety disorders: from surveys to nosology and back. Dialogues Clin Neurosci. 2017; 19:127-136.

2. American Psychiatric Association. Diagnostic and Statistical Manual of Mental Disorders, 5th Edition (DSM-5). Arlington, VA: American Psychiatric Association; 2013.

3. Flores A, Fullana MA, Soriano-Mas C, Andero R. Lost in translation: how to upgrade fear memory research. Mol Psychiatry. 2018;23:2122-2132.

4. Marsicano G, Kuner R. Anatomical distribution of receptors, ligands and enzymes in the brain and in the spinal cord: circuitries and neurochemistry. Cannabinoids Brain. 2008;2:161-201.

5. Lutz B, Marsicano G, Maldonado R, Hillard CJ. The endocannabinoid system in guarding against fear, anxiety and stress. Nat Rev Neurosci. 2015;16:705-718.

6. Shin LM, Liberzon I. The neurocircuitry of fear, stress, and anxiety disorders. Neuropsychopharmacology. 2010;35:169-191.

7. Stahl SM. Stahl's Essential Psychopharmacology Neuroscientific Basis and Practical Application 4th Edition. Cambridge, UK: Cambridge University Press; 2015.

8. Ruehle S, Rey AA, Remmers F, Lutz B. The endocannabinoid system in anxiety, fear memory and habituation. J. Psychopharmacol. 2012;26:23-39.

9. Lutz B. The endocannabinoid system and extinction learning. Mol Neurobiol. 2007;36:92-101.

10. Dubreucq S, Matias I, Cardinal P, et al. Genetic dissection of the role of cannabinoid type-1 receptors in the emotional consequences of repeated social stress in mice. Neuropsychopharmacology. 2012:37:1885-1900.

11. Metna-Laurent M, Soría-Gomez E, Verrier D, et al. Bimodal control of fear-coping strategies by CB 1 cannabinoid receptors. J Neurosci. 2012;32: 7109-7118.

12. Busquets-Garcia A, Desprez T, Metna-Laurent M, Bellocchio L, Marsicano G, Soria-Gomez E. Dissecting the cannabinergic control of behavior: the where matters. Bioessays. 2015;37:1215-1225.

13. Bellocchio L, Lafenêtre $P$, Cannich $A$, et al. Bimodal control of stimulated food intake by the endocannabinoid system. Nat Neurosci. 2010; 13:281-283.

14. Steindel F, Lerner R, Häring $M$, et al. Neuron-type specific cannabinoid-mediated $\mathrm{G}$ protein signalling in mouse hippocampus. J Neurochem. 2013; 124:795-807.

15. Kamprath K, Romo-Parra H, Häring $M$, et al. Short-term adaptation of conditioned fear responses through endocannabinoid signaling in the central amygdala. Neuropsychopharmacology. 2011;36: 652-663.

16. Lin HC, Mao SC, Su CL, Gean PW. The role of prefrontal cortex $\mathrm{CB} 1$ receptors in the modulation of fear memory. Cereb Cortex. 2009;19:165-175.
17. Jenniches I, Ternes S, Albayram O, et al. Anxiety, stress, and fear response in mice with reduced endocannabinoid levels. Biol Psychiatry. 2016;79:858-868. 18. Gunduz-Cinar O, MacPherson KP, Cinar R, et al. Convergent translational evidence of a role for anandamide in amygdala-mediated fear extinction, threat processing and stress-reactivity. Mol Psychiatry. 2013;18:813-823.

19. Ng LK, Lamprecht F, Williams RB, Kopin IJ. $\Delta^{9}$-tetrahydrocannabinol and ethanol: differential effects on sympathetic activity in differing environmental setting. Science. 1973;180:1368-1369.

20. Busquets-Garcia A, Gomis-González M, Srivastava RK, et al. Peripheral and central $\mathrm{CB}_{1}$ cannabinoid receptors control stress-induced impairment of memory consolidation. Proc Natl Acad Sci U S A. 2016;113:9904-9909.

21. Bellocchio L, Soria-Gómez E, Quarta C, et al. Activation of the sympathetic nervous system mediates hypophagic and anxiety-like effects of $\mathrm{CB}_{1}$ receptor blockade. Proc Natl Acad Sci U S A. 2013; 110:4786-4791.

22. Cota D, Marsicano G, Tschöp M, et al. The endogenous cannabinoid system affects energy balance via central orexigenic drive and peripheral lipogenesis. J Clin Invest. 2003;112:423-431.

23. Hill MN, Karatsoreos IN, Hillard CJ, McEwen BS. Rapid elevations in limbic endocannabinoid content by glucocorticoid hormones in vivo. Psychoneuroendocrinology. 2010;35:1333-1338. 24. Herman JP, Ostrander MM, Mueller NK, Figueiredo H. Limbic system mechanisms of stress regulation: hypothalamo-pituitary-adrenocortical axis. Prog Neuropsychopharmacol Biol Psychiatry. 2005; 29:1201-1213

25. Di S, Itoga CA, Fisher MO, et al. Acute stress suppresses synaptic inhibition and increases anxiety via endocannabinoid release in the basolateral amygdala. J Neurosci. 2016;36:8461-8670.

26. Zehra A, Burns J, Liu CK, et al. Cannabis addiction and the brain: a review. $J$ Neuroimmune Pharmacol. 2018;13:438-452.

27. Saravia R, Ten-Blanco M, Julià-Hernández M, et al. Concomitant THC and stress adolescent exposure induces impaired fear extinction and related neurobiological changes in adulthood. Neuropharmacology. 2019;144:345-357.

28. Christensen R, Kristensen PK, Bartels EM, Bliddal H, Astrup A. Efficacy and safety of the weightloss drug rimonabant: a meta-analysis of randomised trials. Lancet. 2007:370:1706-1713.

29. Gray JM, Vecchiarelli HA, Morena M, et al. Corticotropin-releasing hormone drives anandamide hydrolysis in the amygdala to promote anxiety. J Neurosci. 2015:35:3879-3892.

30. Hartmann A, Fassini A, Scopinho A, et al. Role of the endocannabinoid system in the dorsal hippocampus in the cardiovascular changes and delayed anxiety-like effect induced by acute restraint stress in rats. J Psychopharmacol. 2019;33:606-614.
31. Navarria A, Tamburella A, Iannotti FA, et al. The dual blocker of FAAH/TRPV1 N-arachidonoylserotonin reverses the behavioral despair induced by stress in rats and modulates the HPA-axis. Pharmacol Res. 2014;87:151-159.

32. Hill MN, McLaughlin RJ, Pan B, et al. Recruitment of prefrontal cortical endocannabinoid signaling by glucocorticoids contributes to termination of the stress response. $J$ Neurosci. 2011;31: 10506-10515.

33. Morena M, Patel S, Bains JS, Hill MN. Neurobiological interactions between stress and the endocannabinoid system. Neuropsychopharmacology. 2016;41:80-102

34. McEwen BS. Brain on stress: how the social environment gets under the skin. Proc Natl Acad Sci U S A. 2012;109(suppl):17180-17185.

35. Micale V, Drago F. Endocannabinoid system, stress and HPA axis. Eur J Pharmacol. 2018;834: 230-239.

36. Natividad LA, Buczynski MW, Herman MA, et al. Constitutive increases in amygdalar corticotropin-releasing factor and fatty acid amide hydrolase drive an anxious phenotype. Biol Psychiatry. 2017;82: 500-510.

37. Duan T, Gu N, Wang Y, et al. Fatty acid amide hydrolase inhibitors produce rapid anti-anxiety responses through amygdala long-term depression in male rodents. J Psychiatry Neurosci. 2017;42: 230-241.

38. Yasmin F, Colangeli R, Morena M, et al. Stress-induced modulation of endocannabinoid signaling leads to delayed strengthening of synaptic connectivity in the amygdala. Proc Natl Acad Sci US A. 2020;117:650-655.

39. Mitra R, Jadhav S, McEwen BS, Vyas A, Chattarji S. Stress duration modulates the spatiotemporal patterns of spine formation in the basolateral amygdala. Proc Natl Acad Sci U S A. 2005;102: 9371-9376.

40. Wang W, Sun D, Pan B, et al. Deficiency in endocannabinoid signaling in the nucleus accumbens induced by chronic unpredictable stress. Neuropsychopharmacology. 2010;35:2249-2261.

41. Newsom RJ, Stafford J, Garcia RJ, Campeau $\mathrm{S}$. Endocannabinoid signaling as an intrinsic component of the circuits mediating adaptive responses to repeated stress exposure in adult male Sprague Dawley rats. Stress. 2020;23:174-189.

42. Oddi S, Scipioni L, Maccarrone M. Endocannabinoid system and adult neurogenesis: a focused review. Curr Opin Pharmacol. 2019;50:25-32.

43. Marcus DJ, Bedse G, Gaulden AD, et al. Endocannabinoid signaling collapse mediates stress-induced amygdalo-cortical strengthening. Neuron. 2020;105(6):1062-1076.e6. doi:10.1016/j.neuron. 2019.12.024.

44. Liu K. How I faced my coronavirus anxiety. Science. 2020;367:1398.

45. LeDoux JE, Pine DS. Using neuroscience to help 


\section{Original article}

The endocannabinoid system in fear, anxiety, and stress - Maldonado et al understand fear and anxiety: a two-system framework. Am J Psychiatry. 2016;173:1083-1093.

46. Grupe DW, Nitschke JB. Uncertainty and anticipation in anxiety: an integrated neurobiological and psychological perspective. Nat Rev Neurosci. 2013;14:488-501

47. Van Ameringen M, Zhang J, Patterson B, Turna $\mathrm{J}$. The role of cannabis in treating anxiety. Curr Opin Psychiatry. 2020;33(1):1-7.

48. Hall W, Solowij N. Adverse effects of cannabis. Lancet. 1998:352:1611-1616.

49. Rey AA, Purrio M, Viveros MP, Lutz B. Biphasic effects of cannabinoids in anxiety responses: $\mathrm{CB} 1$ and $\mathrm{GABA}_{\mathrm{B}}$ receptors in the balance of GABAergic and glutamatergic neurotransmission. Neuropsychopharmacology. 2012;37:2624-2634.

50. Monory K, Polack M, Remus A, Lutz B, Korte M. Cannabinoid CB1 receptor calibrates excitatory synaptic balance in the mouse hippocampus. $\mathrm{J} \mathrm{Neu}$ rosci. 2015;35:3842-3850.

51. Ruehle $\mathrm{S}$, Remmers F, Romo-Parra $\mathrm{H}$, et al. Cannabinoid CB1 receptor in dorsal telencephalic glutamatergic neurons: distinctive sufficiency for hippocampus-dependent and amygdala-dependent synaptic and behavioral functions. J Neurosci. 2013;33:10264-10277.

52. Lafenêtre P, Chaouloff F, Marsicano G. Bidirectional regulation of novelty-induced behavioral inhibition by the endocannabinoid system. Neuropharmacology. 2009;57:715-721.

53. Häring M, Kaiser N, Monory K, Lutz B. Circuit specific functions of cannabinoid $\mathrm{CB} 1$ receptor in the balance of investigatory drive and exploration. PLoS One. 2011;6:e26617.

54. Fuss J, Steinle J, Bindila L, et al. A runner's high depends on cannabinoid receptors in mice. Proc Natl Acad Sci U S A. 2015;112:13105-13018.

55. Chicca A, Nicolussi S, Bartholomäus R, et al. Chemical probes to potently and selectively inhibit endocannabinoid cellular reuptake. Proc Natl Acad Sci U S A. 2017;114:E5006-E5015.

56. Bedse G, Hartley ND, Neale E, et al. Functional redundancy between canonical endocannabinoid signaling systems in the modulation of anxiety. Biol Psychiatry. 2017;82:488-499.

57. Bedse G, Hill MN, Patel S. 2-Arachidonoylglycerol modulation of anxiety and stress adaptation: from grass roots to novel therapeutics. Biol Psychiatry. 2020;1995:1-11.

58. Hammoud MZ, Peters C, Hatfield JRB, et al.
Influence of $\Delta^{9}$-tetrahydrocannabinol on long-term neural correlates of threat extinction memory retention in humans. Neuropsychopharmacology. 2019;44:1769-1777.

59. Longaretti A, Forastieri C, Gabaglio M, Rubino T, Battaglioli E, Rusconi F. Termination of acute stress response by the endocannabinoid system is regulated through lysine-specific demethylase 1-mediated transcriptional repression of 2-AG hydrolases ABHD6 and MAGL. J Neurochem. March 5, 2020. Epub ahead of print. doi:10.1111/ jnc. 15000 .

60. Lange MD, Daldrup T, Remmers F, et al. Cannabinoid $\mathrm{CB} 1$ receptors in distinct circuits of the extended amygdala determine fear responsiveness to unpredictable threat. Mol Psychiatry. 2017;22: 1422-1430

61. Viñals X, Moreno E, Lanfumey L, et al. Cognitive impairment induced by Delta9-tetrahydrocannabinol occurs through heteromers between cannabinoid $\mathrm{CB}_{1}$ and serotonin 5-HT receptors. PLoS Biol. 2015;13:e1002194.

62. Häring M, Enk V, Rey AA, et al. Cannabinoid type-1 receptor signaling in central serotonergic neurons regulates anxiety-like behavior and sociability. Front Behav Neurosci. 2015;9:235.

63. Geddes SD, Assadzada S, Lemelin D, et al. Target-specific modulation of the descending prefrontal cortex inputs to the dorsal raphe nucleus by cannabinoids. Proc Natl Acad Sci U S A. 2016;113: 5429-5434.

64. Berger AL, Henricks AM, Lugo JM, et al. The lateral habenula directs coping styles under conditions of stress via recruitment of the endocannabinoid system. Biol Psychiatry. 2018;84:611-623.

65. Alteba S, Korem N, Akirav I. Cannabinoids reverse the effects of early stress on neurocognitive performance in adulthood. Learn Mem. 2016;23: 349-358.

66. Bowers ME, Ressler KJ. Sex-dependence of anxiety-like behavior in cannabinoid receptor 1 (Cnr1) knockout mice. Behav Brain Res. 2016; 300:65-69.

67. Simone JJ, Baumbach JL, McCormick CM. Effects of $\mathrm{CB} 1$ receptor antagonism and stress exposures in adolescence on socioemotional behaviours, neuroendocrine stress responses, and expression of relevant proteins in the hippocampus and prefrontal cortex in rats. Neuropharmacology. 2018;128: 433-447.
68. Bara A, Manduca A, Barnabeu A, et al. Sex-dependent effects of in utero cannabinoid exposure on cortical function. Elife. 2018;7:e36234.

69. Cinquina V, Calvigioni D, Farlik M, et al. Lifelong epigenetic programming of cortical architecture by maternal 'Western' diet during pregnancy. Mol Psychiatry. 2020;25:22-36.

70. Pushkin AN, Fingerle V, Oskam C, Stevenson B, Gofton A. Cannabinoid and nicotine exposure during adolescence induces sex-specific effects on anxiety- and reward-related behaviors during adulthood. PLoS One. 2019;14:e211346.

71. Cossio D, Stadler H, Michas Z, Johnston C, Lopez HH. Disrupting the endocannabinoid system in early adolescence negatively impacts sociability. Pharmacol Biochem Behav. 2020;188:172832.

72. Mohammed AN, Alugubelly N, Kaplan BL, Carr RL. Effect of repeated juvenile exposure to $\Delta^{9}$-tetrahydrocannabinol on anxiety-related behavior and social interactions in adolescent rats. Neurotoxicol Teratol. 2018;69:11-20.

73. Bruijnzeel AW, Knight P, Panunzio S, et al. Effects in rats of adolescent exposure to cannabis smoke or THC on emotional behavior and cognitive function in adulthood. Psychopharmacology (Berl). 2019;236:2773-2784

74. Spechler PA, Orr CA, Chaarani B, et al. Cannabis use in early adolescence: evidence of amygdala hypersensitivity to signals of threat. Dev Cogn Neurosci. 2015;16:63-70.

75. Orr C, Spechler P, Cao Z, et al. Grey matter volume differences associated with extremely low levels of cannabis use in adolescence. $J$ Neurosci. 2019;39:1817-1827.

76. Grunberg VA, Cordova KA, Bidwell LC, Ito TA. Can marijuana make it better? Prospective effects of marijuana and temperament on risk for anxiety and depression. Psychol Addict Behav. 2015;29:590-602. 77. Sartori SB, Singewald N. Novel pharmacological targets in drug development for the treatment of anxiety and anxiety-related disorders. Pharmacol Ther. 2019:204:107402.

78. Fowler CJ. The potential of inhibitors of endocannabinoid metabolism for drug development: a critical review. In: Pertwee RG, ed. Handbook of Experimental Pharmacology; vol 231. New York, NY: Springer New York; 2015:95-128.

79. Vallee M, Vitiello S, Bellocchio L, et al. Pregnenolone can protect the brain from cannabis intoxication. Science. 2014;343:94-98. 
